\title{
ADERÊNCIA DE MICROORGANISMOS EM MATERIAIS PARA BASE DE PRÓTESES
}

\section{ADHERENCE OF MICROORGANISMS TO DENTURE BASE MATERIALS}

\author{
Karin Hermana Neppelenbroek * \\ Elaine Cristina Tavares Pinto ** \\ Ana Cláudia Pavarina *** \\ Carlos Eduardo Vergani *** \\ Janaina Habib Jorge ***** \\ Hercules Jorge Almilhatti
}

\section{RESUMO}

A aderência de microrganismos nos materiais para base de prótese pode favorecer o desenvolvimento de doenças como estomatite protética, cáries e doenças periodontais nos usuários de próteses removíveis parciais e totais. As características da placa microbiana presente nas próteses podem ser influenciadas por fatores relacionados ao paciente, como idade, sexo, condiçóes sistêmicas, qualidade da microbiota bucal e uso de medicamentos e por fatores relacionados à prótese como condiçóes de estado (suporte, retenção e estabilidade), tempo de utilização, métodos de higienização, propriedades físico-químicas da resina acrílica utilizada para a confecção da base e técnicas de confecção e polimento da prótese. Este estudo tem por objetivo discutir, por meio de uma revisão de literatura, os fatores associados à aderência de microrganismos aos materiais utilizados para a confecção de base de próteses.

DESCRITORES: Prótese parcial removível • Prótese total • Aderência celular • Resinas acrílicas

\section{ABSTRACT}

The adherence of microorganisms on denture base materials may help determine the development of diseases such as denture stomatitis, caries e periodontal diseases in complete and partial denture users. The features of the denture plaque may be influenced by factors related to the patient such as gender, age, systemic conditions, quality of oral environment and use of medication, and factors related to denture such as conditions of support, retention, and stability, denture age, hygiene practices, chemical and physical properties of denture base resin and techniques for fabrication and polishing denture bases. This study aimed to discuss, by a literature review, the factors related to the adherence of microorganisms on the denture base materials.

DESCRIPTORS: Denture partial removable $\bullet$ Denture, complete $\bullet$ Cell adhesion $\bullet$ Acrylic resins

\footnotetext{
* Professor Doutor das Disciplinas de Prótese Total I e II e Clínica Integrada Reabilitadora I, Departamento de Prótese, Faculdade de Odontologia de Bauru - USP

** Especialista em Reabilitaçáo Oral, Departamento de Materiais Odontológicos e Prótese, Faculdade de Odontologia de Araraquara - UNESP

*** Professor Adjunto da Disciplina de Prótese Parcial Removível, Departamento de Materiais Odontológicos e Prótese, Faculdade de Odontologia de Araraquara - UNESP

**** Professor Adjunto da Disciplina de Oclusão, Setor de Ciências Biológicas e da Saúde, Departamento de Odontologia, Universidade Estadual de Ponta Grossa.

***** Professor Adjunto da Disciplina de Prótese Fixa, Departamento de Odontologia Restauradora da Universidade Federal do Paraná
} 
Neppelenbroek KH, Pinto ECT, Pavarina AC, Vergani CE, Jorge JH, Almilhatti HJ. Aderência de microorganismos em materiais para base de próteses Revista de Odontologia da Universidade Cidade de São Paulo 2009 mai-ago; 21(2): 126-36

\section{INTRODUÇÃO}

Além do restabelecimento da função mastigatória e da estética, a reabilitação de pacientes total ou parcialmente desdentados com próteses removíveis tem como objetivo a preservação das estruturas bucais remanescentes. No entanto, durante o período de utilização, as próteses removíveis permanecem frequentemente em contato com os fluidos bucais. A presença de alta concentração de proteína nos fluidos bucais permite a formação de uma película sobre a superfície das próteses, proporcionando condiçóes favoráveis à aderência de microrganismos tanto nessas superfícies quanto nos tecidos bucais que mantêm contato com as próteses (Gristina et al. ${ }^{15}$ 1987). A aderência de microrganismos aos materiais que constituem as próteses e aos tecidos bucais é um fator determinante para a colonização microbiana e consequentemente para o desenvolvimento de problemas associados à utilização de próteses removíveis, totais ou parciais (Nikawa et al. ${ }^{28}$ 1998, Sato et al. ${ }^{40}$ 1997). Entre essas alterações podem ser citadas doenças gengivais e periodontais e cáries dentais, principalmente envolvendo as regióes radiculares e infecções como a estomatite protética nos tecidos bucais adjacentes às bases das próteses (Nikawa et al. ${ }^{28} 1998$, Radford et al. ${ }^{37}$ 1999). As leveduras do gênero Candida são comumente encontradas na placa presente nas próteses de resina acrílica Nikawa et al..$^{28} 1998$, Sato et al. ${ }^{40}$ 1997). No entanto, outras espécies de microrganismos também têm sido consideradas como importantes patógenos na formação e manutenção da placa associada a essas próteses (Cross et al. ${ }^{13}$ 2004, Morgan e Wilson ${ }^{24}$ 2001, Peres et al..$^{32}$ 2002, Sato et al..$^{40} 1997$, Thelaide e Budtz-Jorgensen ${ }^{48} 1998$, Willis et al. ${ }^{56} 2000$ ).

Apesar da aderência de microrganismos poder ser verificada em todas as superfícies de uma prótese removível, as superfícies internas das bases de resina acrílica frequentemente apresentam a maior quantidade de placa microbiana (Quirinem e Bollen ${ }^{35} 1995$, Radford et al. ${ }^{38}$ 1998). Essas superfícies não são polidas, apresentando maior quantidade de irregularidades o que favorece a aderência de microrganismos (Quirinem e Bollen ${ }^{35}$ 1995, Sato et al..$^{40}$ 1997). As características da placa microbiana presente nas próteses podem ser influenciadas por fatores relacionados ao paciente, como idade, sexo, condiçóes sistêmicas, qualidade da microbiota bucal e uso de medicamentos e por fatores relacionados à prótese como condiçôes de estado (suporte, retenção e estabilidade), tempo de utilização, métodos de higienização, propriedades físico-químicas da resina acrílica utilizada para a confecção da base e técnicas de confecção e polimento da prótese (Budtz-Jorgensen ${ }^{7}$ 1978, Massad e Cagna $^{21}$ 2002, Neppelenbroek et al. ${ }^{26}$ 2008, Nikawa et al. ${ }^{28}$ 1998, Pfaller e Welzel ${ }^{31} 1992$, Pittet el al..$^{33} 1993$, Quirinen et al..$^{36}$ 1990, Radford et al. ${ }^{38}$ 1998, Sipahi et al. ${ }^{44}$ 2001, Verran e Marian ${ }^{52}$ 1997, Willis et al. ${ }^{56} 2000$, Yamauchi et al. ${ }^{58}$ 1990). Como a maior quantidade de placa microbiana é encontrada nas superfícies internas das bases das próteses que mantêm contato com os tecidos bucais, é importante considerar, quando na confecçâo de próteses removíveis, totais ou parciais, os fatores associados à aderência de microrganismos nessas superfícies (Quirinem e Bollen ${ }^{35}$ 1995). Dessa forma, é possível reduzir a possibilidade de formação de placa nas próteses e consequentemente favorecer a saúde bucal do paciente. (Barnabé et al. ${ }^{4}$ 2004). Entre os fatores associados à aderência de microrganismos nos materiais para base de prótese estão a composição e a forma de polimerização da resina acrílica (Nepellenbroek et al..$^{25}$ 2006, Nepellenbroek et al. ${ }^{27}$ 2003). Esses fatores podem influenciar diretamente as características superficiais das resinas acrílicas que são consideradas importantes na determinação da aderência microbiana, tais como rugosidade superficial e energia livre de superfície (Tari et al. ${ }^{47}$ 2007, Van Pelt et al. ${ }^{51} 1985$, Yamauchi et al..$\left.^{58} 1990\right)$. Tendo em vista as consideraçóes anteriormente citadas, este estudo tem por objetivo discutir, por meio de uma revisão de literatura, os fatores associados à aderência de microrganismos aos materiais para base de próteses.

\section{REVISÃO DA LITERATURA}

O processo de aderência nas bases de resina acrílica ocorre em quatro fases: os microrganismos presentes nos fluidos bucais atingem a película formada sobre a prótese, principalmente na superfície que mantém contato com o rebordo residual; os microrganismos aderem ao tecido bucal e à prótese, por um processo de adesão inicial que pode ser reversível ou irreversível; os microrganismos são fixados nas células epiteliais e na resina acrílica por meio de interaçóes especificas; ocorre então os processos de saturação e colonização microbiana e consequentemente uma fina camada de biofilme é formada (Quirinem e Bollen $^{35}$ 1995, Sato et al..$^{40}$ 1997). O aumento da espessura e da complexidade da composição da camada de biofilme propicia a formação da placa microbiana. A placa presente nas próteses com base de resina acrílica é considerada um biofilme complexo, formado por leveduras, bactérias e células epiteliais descamativas (Neppe- 
Neppelenbroek KH, Pinto ECT, Pavarina AC, Vergani CE, Jorge JH, Almilhatti HJ. Aderência de microorganismos em materiais para base de próteses Revista de Odontologia da Universidade Cidade de São Paulo 2009 mai-ago; 21 (2): 126-36

lenbroek et al. ${ }^{26}$ 2008, Sato et al. $\left.{ }^{40} 1997\right)$. As formas mais comuns de bactérias isoladas de biofilmes presentes nas próteses são os bacilos e os cocos, especialmente as espécies de Streptococcus, Staphylococcus, Bacteroides, Neisseria, Lactobacillus e Actinomyces (Barnabé et al. ${ }^{4} 2004$, Kulak et al. ${ }^{18}$ 1997, Baena-Monroy et al. ${ }^{3}$ 2005, Morgan e Wilson ${ }^{24}$ 2001, Sato et al. ${ }^{40}$ 1997). Essas bactérias vêm sendo relatadas como importantes patógenos na formação e manutenção da placa microbiana associada às bases de resina acrílica. Tem sido sugerido que as bactérias possivelmente favorecem a adesão de leveduras do gênero Candida às superfícies internas das próteses por coagregação (Sato et al. ${ }^{40}$ 1997). As leveduras do gênero Candida, principalmente $C$. albicans, estão comumente presentes na placa microbiana associada às próteses removíveis totais e parciais (Kulak et al. ${ }^{18}$ 1997, Nikawa et al. ${ }^{28}$ 1998, Radford et al. ${ }^{37}$ 1999). Ainda, outras espécies menos patogênicas que a $C$. albicans, tais como $C$. glabrata, C. tropicalis, C. guilliermondii, C. parapsilosis, $C$. dubliniensis e $C$. krusei, têm sido recentemente isoladas das bases acrílicas das próteses (Cross et al. ${ }^{13}$ 2004, Peres et al. ${ }^{32}$ 2002). A placa microbiana presente nas próteses, sobretudo quando associada à presença das leveduras do gênero Candida, é considerada o maior fator etiológico da estomatite protética (Budtz-Jorgensen ${ }^{7}$ 1978, Budtzjorgensen $^{8}$ 1979, Dovenport ${ }^{14}$ 1970, Nikawa et al. ${ }^{28}$ 1998). Os microrganismos presentes na placa podem penetrar na resina acrílica das bases de próteses e sobreviver a uma profundidade que varia de 1,0 a 2,0 micrômetros (Chau et al. ${ }^{11}$ 1995). Assim, mesmo quando há controle da placa microbiana, é possível que os microrganismos não sejam eliminados nas regióes mais internas da resina acrílica, causando reinfecção da mucosa do paciente via prótese (Dovenport ${ }^{14}$ 1970). Além disso, a colonização bucal promovida pelas superfícies das próteses removíveis, principalmente pelas superfícies da regiáo interna, pode funcionar como um reservatório de infecções tais como as gastrointestinais (Gristina et al. ${ }^{15}$ 1987). O tipo de microrganismo que pode ser disseminado pela resina acrílica varia de acordo com o acúmulo e a composição da placa microbiana presente nas superfícies das próteses (Chau et al. ${ }^{11}$ 1995). Cabe ao profissional orientar o paciente quanto aos métodos de limpeza e desinfecção da prótese que são efetivos na remoção da placa microbiana e na inativação dos microrganismos colonizadores da resina acrílica. Apesar da escovação com dentifrício ser o método mais utilizado para limpeza das próteses, tem sido demonstrado que esse procedimento é insuficiente para um controle efetivo do biofilme presente nas bases acrílicas das próteses removíveis (Barnabé et al. ${ }^{4} 2004$, Budtz-Jorgensen ${ }^{8}$ 1979, Chau et al. ${ }^{11}$ 1995, Kulak et al. ${ }^{18}$ 1997). A associaçáo de outros métodos como imersão noturna em solução de hipoclorito de sódio 0,02\% (Webb et al. ${ }^{54}$ 2005) e clorexidina a $1 \%$ (Kulak et al ${ }^{18}$ 1997), vem sendo recomendada. Como essas soluçóes podem causar manchamento à resina acrílica, tem sido sugerido como método efetivo de inativação microbiana a associaçáo de escovação seguida por um protocolo de desinfecção em microondas previamente determinado por estudos in vitro (Nepellenbroek et al. ${ }^{27}$ 2003, Silva et al. ${ }^{43}$ 2006), e in vivo (Neppelenbroek et al. ${ }^{26}$ 2008).

Entre os fatores associados à aderência de microrganismos nos materiais para base de prótese estão a composição da resina acrílica e a sua técnica de polimerização. Foi demonstrado que as resinas termopolimerizáveis polimerizadas por microondas apresentam adaptação e propriedades mecânicas semelhantes às apresentadas para as resinas termopolimerizáveis polimerizadas em banho de água (Smith et al. ${ }^{45}$ 1992). Também foi verificado que esses materiais não apresentaram diferenças estatisticamente significante em relação à porosidade (Compagnoni et al. ${ }^{12}$ 2004). Devido à similaridade apresentada entre as resinas termopolimerizáveis por microondas e por banho de água, principalmente em relaçáo à porosidade, é possível supor que não haja diferença significante entre a aderência de microrganismos nesses materiais. No entanto, é necessário respaldo científico para confirmação dessa hipótese, uma vez que não foram encontrados na literatura disponível estudos que comparassem a aderência de microrganismos entre esses materiais. As análises por meio de microscopia eletrônica de varredura realizadas no estudo de Kuhar e Funduk ${ }^{17}$ (2005), evidenciaram maior porosidade superficial em uma resina autopolimerizável em relação às duas resinas termopolimerizáveis avaliadas. A presença de poros aumenta o acúmulo de placa na superfície da resina acrílica e o manchamento de algumas partes das próteses (Bunch et al. ${ }^{9}$ 1987, Chau et al. ${ }^{11}$ 1995). Uma vez aderidos em um biofilme à superfície de uma resina acrílica com maior porosidade, leveduras e bactérias se tornam protegidas contra as forças de cisalhamento da limpeza mecânica (Baena-Monroy et al. ${ }^{3}$ 2005). Consequentemente, as resinas acrílicas autopolimerizáveis podem ser mais facilmente colonizadas e profundamente infectadas por microrganismos que as resinas termopolimerizáveis (Bunch et al. ${ }^{9}$ 1987). As resinas acrílicas autopolimeri- 
Neppelenbroek KH, Pinto ECT, Pavarina AC, Vergani CE, Jorge JH, Almilhatti HJ. Aderência de microorganismos em materiais para base de próteses Revista de Odontologia da Universidade Cidade de São Paulo 2009 mai-ago; 21(2): 126-36

záveis rígidas, utilizadas para reembasamento definitivo, apresentam melhores propriedades físico-químicas que as resinas autopolimerizáveis convencionais (Arima et al. ${ }^{2}$ 1995). Tem sido demonstrado que esses materiais apresentam aderência microbiana semelhante à apresentada por resinas termopolimerizáveis. Neppelenbroek et $a .^{25}$ (2006) observaram que não houve diferença significativa entre duas resinas reembasadoras rígidas e uma resina termopolimerizável quanto à aderência de dois microrganismos patogênicos (Staphylococcus aureus e Pseudomonas aeruginosa). Por outro lado, a resina termopolimerizável apresentou maior aderência de $C$. albicans em relação às resinas rígidas avaliadas. Os resultados favoráveis de baixa aderência microbiana em materiais reembasadores rígidos têm sido atribuídos à estrutura polimérica determinada pela alta concentração de agentes de ligação cruzada e pela presença de monômeros bi-funcionais (Arima et al.2 1995, Nepellenbroek et al. ${ }^{25} 2006$, Nepellenbroek et al. ${ }^{27}$ 2003). As resinas do tipo "soft" ou macias, utilizadas para reembasamento temporário, são consideradas os materiais para base de prótese mais propícios à aderência microbiana que as resinas termopolimerizáveis e às resinas reembasadoras rígidas. Radford et al. ${ }^{37}$ (1999) verificaram que os materiais reembasadores macios avaliados apresentaram maior aderência de $C$. albicans que a resina termopolimerizável testada. Como são capazes de interagir com os microrganismos bucais devido à sua textura superficial e características físicoquímicas, os materiais reembasadores macios favorecem a retenção microbiana (Baysan et al. ${ }^{5}$ 1998, Waters et al. ${ }^{53}$ 1997).

A aderência de microrganismos às superfícies de resina acrílica das bases das próteses também pode ser influenciada pelo tipo de microrganismo presente na placa. Verran e Maryan 52, 1997 relataram que células maiores, como as leveduras, são facilmente deslocadas da superfície da resina acrílica quando em comparação com células menores como as bacterianas. Essas diferenças foram observadas por Neppelenbroek et al. ${ }^{25}$ 2006, Nepellenbroek et al. ${ }^{27} 2003$, que concluíram que a aderência de duas espécies de bactérias ( $S$. aureus e $P$. aeruginosa) às superfícies de 5 reembasadores rígidos e uma resina para base de prótese foi superior à aderência de levedura de $C$. albicans. Assim, é provável que a aderência entre os microrganismos colonizadores de uma base acrílica varie entre os diferentes tamanhos e tipos de células sendo também influenciada pelos mecanismos de adesão apresentados por cada espécie. Assim, dependendo da qualidade da microbiota do paciente, é possível haver prevalência de uma espécie sobre a outra, o que irá determinar a susceptibilidade do paciente a uma dada patologia.

As propriedades relacionadas às superfícies dos materiais utilizados para a confecção de próteses são importantes do ponto de vista clínico, uma vez que podem interferir com o acúmulo de placa e manchamento. Vários estudos têm demonstrado que as superfícies de resina acrílica com maior rugosidade superficial são significativamente mais propícias à aderência microbiana que as superfícies mais polidas, ou seja, com menor rugosidade superficial (Hannig ${ }^{16}$ 1999, Morgan e Wilson ${ }^{24}$ 2001, Quirinen et al. ${ }^{36}$ 1990, Radford et al. ${ }^{38} 1998$, Zissis et al. ${ }^{59}$ 2000). A adesão microbiana nas superfícies internas das próteses merece significativa atenção uma vez que essas superfícies não são polidas para manter adequada adaptação aos tecidos de suporte. Existem importantes diferenças entre a microbiota observada nas superfícies de suporte e aquela presente nas superfícies externas das próteses. Assim como nas regióes subgengivais dos dentes naturais, o espaço sob a superfície de suporte das próteses funciona como um sistema fechado que favorece a adesão e colonização microbiana (Quirynen e Bollen ${ }^{35}$, 1995). Sob esse espaço, fungos e bactérias colonizam a superfície de suporte das próteses e a mucosa epitelial do paciente. Essas colônias podem tanto flutuar livremente em fina camada de saliva quanto penetrar nos tecidos mucosos do paciente e nas regióes profundas dos poros da resina acrílica (Chau et al. ${ }^{11} 1995$, Monsenego $^{22}$ 2000, Nikawa et al. ${ }^{28} 1998$, Radford et al. ${ }^{37}$ 1999). Nesse ambiente, o impacto da rugosidade superficial na adesão microbiana se torna muito menor. Entretanto, a importância da rugosidade superficial nas superfícies de suporte das próteses aumenta sempre que a prótese é higienizada pelo paciente. (Monsenego ${ }^{22}$ 2000, Morgan e Wilson ${ }^{24}$ 2001). O polimento das superfícies das próteses que não mantém contato com os tecidos de suporte (superfície externa da base acrílica e dentes artificiais) é um aspecto que também deve considerado pelo profissional. Como a rugosidade superficial dessas superfícies favorece a adesão microbiana e consequentemente a formação de placa, é importante que o polimento das próteses em laboratório seja efetivo de forma a propiciar lisura superficial adequada (Braun et al. ${ }^{6}$ 2003, Hannig ${ }^{16}$ 1999, Quirinen et al. ${ }^{36}$ 1990). Tem sido demonstrado que a rugosidade superficial das resinas acrílicas polidas com pastas profiláticas, pontas de borracha, gessos abrasivos e pedra-pomes geralmente excedem ao limiar abaixo do 
Neppelenbroek KH, Pinto ECT, Pavarina AC, Vergani CE, Jorge JH, Almilhatti HJ. Aderência de microorganismos em materiais para base de próteses Revista de Odontologia da Universidade Cidade de São Paulo 2009 mai-ago; 21 (2): 126-36

qual a aderência de fungos e bactérias não é possível $(0,2$ $\mu \mathrm{m})$ (Loney et al. ${ }^{20} 1994$, Yamauchi et al. ${ }^{58} 1990$ ). Menores valores de rugosidade superficial podem ser obtidos com pasta abrasiva à base de óxido de alumínio ou sabão (Lombardi e Budtz-Jorgesen ${ }^{19}$ 1993, Radford et al. ${ }^{38}$ 1998, Sofou et al. $\left.{ }^{46} 2001\right)$. O polimento convencional em laboratório, geralmente realizado em torno com água e pedra-pomes de granulação fina ou pontas de silicone seguido por pasta ou líquido polidor contendo partículas de óxido de alumínio, tem se mostrado efetivo em reduzir os valores de rugosidade superficial das bases de resinas acrílicas aos limites adequados (Yamauchi $e t$ al. ${ }^{58}$ 1990). No entanto, após a colocação das próteses removíveis, é comum a necessidade de ajustes tanto na superfície de resina acrílica quanto nos dentes artificiais e, para reduzir o tempo clínico, o próprio cirurgião-dentista frequentemente realiza o polimento dessas regióes. Como consequência desse procedimento, vários kits de polimento têm sido introduzidos no mercado odontológico. O estudo de O'Donnell et al. ${ }^{30}$ (2003) demonstrou que 2 kits de polimento para consultório produziu superfícies mais lisas em uma resina termopolimerizável que brocas carbide de tungstênio, ainda que as superfícies com menor rugosidade superficial tenham sido obtidas com o polimento convencional em laboratório. Esses resultados foram semelhantes aos conseguidos por Kuhar e Funduk ${ }^{17}$ (2005) que concluíram que o polimento convencional em laboratório promoveu nas resinas acrílicas avaliadas superfícies significativamente mais polidas (médias de rugosidade inferiores a $0,2 \mu \mathrm{m}$ ) que três kits de polimento com pontas de silicone para consultório que produziram, por sua vez, superfícies mais lisas em relação às brocas carbide de tungstênio. Verran e Maryan ${ }^{52}$ (1997) avaliaram a retenção de $C$. albicans em superfícies lisas e rugosas de resina acrílica termopolimerizável e de silicone para determinar o efeito da rugosidade na infecção das próteses e na higienização. Os resultados obtidos foram de 0,02 e 1,26 $\mu \mathrm{m}$ para as superfícies das resinas acrílicas lisas e rugosas, respectivamente. Zissis et al. ${ }^{59}$ (2000) avaliaram a rugosidade de 9 resinas para base de prótese, 9 materiais reembasadores rígidos e 57 materiais reembasadores macios e concluíram que todos os materiais poderiam apresentar acúmulo de placa, já que a menor rugosidade observada $(0,7 \mu \mathrm{m})$ foi considerada significativamente superior a $0,2 \mu \mathrm{m}$. Os autores também observaram que as resinas termopolimerizáveis para base de prótese exibiram valores médios de 3,4 a $7,6 \mu \mathrm{m}$, enquanto aquelas indicadas para reembasamen- to imediato apresentaram valores médios de 0,7 a 4,4 $\mu \mathrm{m}$. Neppelenbroek et al. ${ }^{25}$ (2006) observaram maior aderência de $C$. albicans na resina termopolimerizável quando comparada às duas resinas reembasadoras rígidas avaliadas, sugerindo que a rugosidade superficial da resina termopolimerizável provavelmente tenha favorecido a aderência. Por outro lado, Kuhar e Funduk ${ }^{17}$ (2005) observaram que os valores de rugosidade superficial de uma resina autopolimerizável foram significativamente superiores aos obtidos com duas resinas termopolimerizáveis, quando submetidas ao mesmo tratamento superficial.

Estudos in vivo e in vitro (Busscher et al. ${ }^{10} 1984$, Van Pelt et al. ${ }^{51} 1985$, Weerkamp et al. ${ }^{55}$ 1985), têm sugerido que existe uma associação entre a energia livre de superfície do substrato e a adesão microbiana. Existem duas explicaçóes para a menor formação de placa microbiana nos substratos com baixa energia livre de superfície: a baixa força de adesão entre o microrganismo e o substrato com baixa energia livre de superfície e a seletividade da adesão microbiana. Segundo Van Pelt et al. ${ }^{51}$ (1985) a energia livre de superfície em um sólido é mais diretamente relacionada à força de adesão de um microrganismo que ao número de microrganismos por área de superfície. Isso foi confirmado por Quiryen et al. ${ }^{34}$ (1989) que observaram fraca força de adesão da placa dental nas superfícies com baixa energia livre de superfície. A seletividade da adesão microbiana é fundamentada na fórmula de Absolom et al. ${ }^{1}$ (1983) na qual a energia livre interfacial de adesão foi correlacionada com as energias livres interfaciais dos tipos sólida, sólido-líquida e líquida de um microrganismo. Com base nessa fórmula, tem sido evidenciado que os microrganismos com baixa energia de superfície aderem preferencialmente às superfícies sólidas com baixa energia e os com alta energia de superfície aderem em maior número nas superfícies com alta energia. Tem sido sugerido que a energia livre de superfície original de um substrato influencia predominantemente a adesão microbiana (Quirinen et al. ${ }^{36} 1990$, Schakenraad et al. $\left.{ }^{41} 1986\right)$. É possível que a própria película adquirida inicialmente funcione como um meio de informação de transferência, o que poderia ser atribuído às diferenças na conformação das moléculas absorvidas, diferenças nos tempos do processo de absorção ou a uma combinação desses processos. Outras explicações têm sido relacionadas às diferenças na quantidade de moléculas absorvidas e às diferenças na cobertura superficial, ou seja, a presença de um filme contínuo ou de padrão irregular (Quirinen et al. ${ }^{36}$ 1990, Schakenraad et al. ${ }^{41}$ 
Neppelenbroek KH, Pinto ECT, Pavarina AC, Vergani CE, Jorge JH, Almilhatti HJ. Aderência de microorganismos em materiais para base de próteses Revista de Odontologia da Universidade Cidade de São Paulo 2009 mai-ago; 21(2): 126-36

1986). Contudo, outros estudos têm proposto que a rugosidade superficial exerce um papel significativamente mais importante na aderência microbiana que a energia livre de superfície (Hannig ${ }^{16}$ 1999, Quirinen e Bollen ${ }^{35}$ 1995, Quirinen et al. ${ }^{36}$ 1990).

Outro fator que pode interferir com a aderência de microrganismos é a umectabilidade do substrato. Monsenego et al. ${ }^{23}$ (1989) relataram que a umectabilidade é medida pelo ângulo de contato do substrato, que é constante para os sólidos e líquidos. Entretanto, o ângulo de contato das resinas acrílicas das próteses em função sofre influência da rugosidade superficial, contaminação da superfície por água e impurezas e reorganização das cadeias poliméricas ocasionada pela migração do monômero residual. $\mathrm{O}$ metil metacrilato é considerado um material de baixa umectabilidade, entretanto, segundo Umemoto e Kurata ${ }^{50}$ (1997) os monômeros hidrofílicos têm maior afinidade aos tecidos mucosos quando comparados aos hidrofóbicos. Tem sido relatado que as resinas termopolimerizáveis apresentam menor ângulo de contato do que as resinas autopolimerizáveis. Quirynen et al. ${ }^{35}$ concluíram que as resinas termopolimerizáveis apresentam maior umectabilidade que as resinas para reembasamento macias à base de silicone. É provável que nas superfícies com maior umectabilidade ocorra maior absorção de proteínas salivares, facilitando dessa forma a aderência microbiana. Entretanto, tem sido observado que as resinas reembasadoras à base de silicone apresentam maior aderência em relação às resinas termopolimerizáveis, o que sugere que a umectabilidade do substrato influencie de forma menos significativa o processo de aderência. A presença de agentes de ligação cruzada também pode alterar essa característica (Umemoto e Kurata $^{50}$ 1997). São necessários novos estudos para avaliar a influência da umectabilidade das resinas acrílicas para bases de próteses na aderência microbiana.

Nikawa et al. ${ }^{28}$ (1998) concluíram que a natureza das proteínas salivares unidas às resinas acrílicas apresenta um papel mais importante na aderência de microrganismos do que as características superficiais dos materiais. Assim, é necessário considerar a qualidade da microbiota do paciente a qual é influenciada por diversas condiçóes, como dieta, qualidade da saliva, condiçóes sistêmicas e uso de medicamentos. A dieta rica em carboidratos cariogênicos (glicose e sacarose) favorece a aderência de microrganismos em relação aos carboidratos não cariogênicos (Nikawa et al. ${ }^{28}$ 1998). Ainda, como a função mastigatória é significativamente reduzida pelo uso de próteses removíveis, os usuários frequentemente apresentam deficiências de importantes nutrientes como ferro, vitamina B e ácido fólico (Ship et al. ${ }^{42}$ 2002). Essas deficiências podem levar à redução da capacidade imunológica do indivíduo, favorecendo o desenvolvimento de infecção por microrganismos oportunistas (BudtzJorgensen ${ }^{7}$ 1978, Pfaller e Welzel ${ }^{31}$ 1992, Pittet el al. ${ }^{33}$ 1993).

Não há, ainda, um consenso para explicar por que várias lesōes bucais causadas por patógenos bucais são mais frequentes em mulheres do que em homens. Essa diferença tem sido atribuída ao fato de as mulheres recorrerem mais frequentemente a tratamentos médicos e odontológicos que os homens (Peres et al. ${ }^{32}$ 2002). Ainda, as mulheres, por razóes estéticas, utilizam suas próteses removíveis por períodos mais longos que os homens. Outro fator está associado às alteraçôes hormonais, principalmente no período após a menopausa (Ritchie et al..$^{39}$ 1969). Com a menopausa, ocorre atrofia da mucosa bucal concomitantemente à redução da secreção hormonal ovariana. Isso torna o epitélio bucal menos resistente a fatores irritantes, favorecendo o desenvolvimento de processos infecciosos (Ritchie et al..$^{39}$ 1969). Indivíduos com xerostomia também são mais susceptíveis à colonizaçâo microbiana nas próteses e mucosa bucal. Essa alteração é observada principalmente em idosos, em decorrência de doenças autoimunes (Síndrome de Sjögren), alteraçóes endócrinas, uso de medicamentos indutores de hiposalivação (anti-hipertensivos, ansiolíticos e hipoglicemiantes) e terapias antineoplásicas de cabeça e pescoço. (Massad e Cagna ${ }^{21}$ 2002, Ship et al. ${ }^{42} 2002$, Torres et al. ${ }^{49}$ 2002). Além da ação imunológica e de limpeza, a saliva ainda protege os tecidos de traumas mecânicos. A xerostomia pode dificultar a retenção das próteses totais, causando traumas aos tecidos e prejuízos às funçōes (Ship et al. ${ }^{42}$ 2002). Tem sido sugerido que os componentes da saliva, tais como mucina, fibrinogênio e anticorpos, podem dificultar a adesão de microrganismos (Massad e Cagna ${ }^{21}$ 2002, Torres et al. ${ }^{49}$ 2002). Outras doenças que comprometam o sistema imunológico também favorecem a proliferação e aderência de microrganismos patogênicos na cavidade bucal e próteses dos pacientes (Pfaller e Welzel ${ }^{31}$ 1992, Pittet el al. ${ }^{33} 1993$, Willis et al. ${ }^{56} 2000$, Wingard ${ }^{57}$ 1995). A utilização das próteses continuamente, inclusive durante o sono, também favorece a colonização microbiana, já que a salivação é significativamente reduzida nesse período (Massad e Cagna ${ }^{21}$ 2002, Nikawa et al. ${ }^{28}$ 1998). O uso contínuo 
Neppelenbroek KH, Pinto ECT, Pavarina AC, Vergani CE, Jorge JH, Almilhatti HJ. Aderência de microorganismos em materiais para base de próteses Revista de Odontologia da Universidade Cidade de São Paulo 2009 mai-ago; 21(2): 126-36

das próteses, principalmente das totais superiores, pode promover traumas aos tecidos de suporte. A pressão negativa sob uma prótese total superior bem adaptada e com efetivo selado periférico pode traumatizar a mucosa de suporte mesmo sem a presença de outro fator predisponente (Budtz-Jorgensen ${ }^{8}$ 1979, Lombardi e BudtzJorgesen $^{19}$ 1993). Além disso, os ductos das glândulas salivares palatinas menores podem ser parcial ou totalmente ocluídos por esse tipo de prótese, o que facilita a colonização microbiana (Ship et al. ${ }^{42}$ 2002).

A reabsorção fisiológica do rebordo causa a perda de adaptação das bases de resina acrílica das próteses à mucosa de suporte, o que resulta em traumas à mucosa de suporte e reabsorção óssea acelerada (Lombardi e BudtzJorgesen ${ }^{19}$ 1993, Ship et al. ${ }^{42}$ 2002). O trauma contínuo pela prótese mal adaptada favorece o desenvolvimento de um processo inflamatório na mucosa de suporte que se torna menos resistente às infecçóes. Por isso, as próteses devem ser periodicamente avaliadas quanto à necessidade de reembasamento e/ou substituição. Após um período de cinco anos, as próteses geralmente apresentam deterioração das superfícies de resina acrílica (desgastes, manchamento, porosidades e trincas), o que favorece o acúmulo de placa microbiana e subsequente infecção (Lombardi e Budtz-Jorgesen ${ }^{19}$ 1993, Ritchie et al. ${ }^{39}$ 1969).

\section{DISCUSSÃO}

As características da placa microbiana presente nas próteses podem ser influenciadas por fatores relacionados ao paciente e à prótese. Como as superfícies das próteses que mantêm contato com os tecidos bucais não são polidas, apresentam maior quantidade de irregularidades e, dessa forma, maior propensão ao acúmulo de placa microbiana (Monsenego ${ }^{22}$ 2000, Quirinem e Bollen ${ }^{35}$ 1995). Assim, quando da confecção de próteses removíveis, totais ou parciais, os fatores associados à aderência de microrganismos nessas superfícies, devem ser considerados. O tipo de material utilizado na confecção das bases das próteses apresenta significativa influência sobre a aderência de microrganismos. Tem sido relatado que as resinas acrílicas termopolimerizáveis, tanto polimerizadas por microondas quanto por banho de água, apresentam menor porosidade em relação às resinas autopolimerizáveis, prevenindo a aderência de microrganismo (Bunch et al. ${ }^{9}$ 1987, Kuar e Funduk ${ }^{17}$ 2005). No entanto, muitas vezes, as próteses removíveis necessitam de reembasamento ou reparo para continuarem de for- ma adequada em função. Normalmente, esses procedimentos são realizados com resinas autopolimerizáveis que passam, dessa forma, a entrar em contato com os tecidos bucais e propiciar maior formação de placa. Os materiais reembasadores macios, por suas propriedades físico-químicas e textura de superfície são os mais favoráveis à aderência de microrganismos (Baysan et al. ${ }^{5} 1998$, Radford et al. ${ }^{38} 1998$, Waters et al. ${ }^{53}$, 1997) Dessa forma, o reembasamento com esse tipo de material deve ser temporário, havendo necessidade contínua de substituição até a confecção de novas próteses. No caso de reparos e reembasamentos definitivos, é possível utilizar resinas autopolimerizáveis com formulações especiais, que possam conferir um desempenho semelhante às resinas termopolimerizáveis (Arima et al. ${ }^{2} 1995$, Bunch et al. ${ }^{9}$ 1987). A alta concentração de agente de ligação cruzada e a presença de monômero bi-funcionais nesses materiais podem minimizar a aderência microbiana em relação às resinas autopolimerizáveis convencionais (Arima et al. ${ }^{2}$ 1995, Nepellenbroek et al. ${ }^{25}$ 2006, Nepellenbroek et al. ${ }^{27}$ 2003).

As características superficiais de uma resina acrílica podem também interferir com a aderência de microrganismos. A influência da rugosidade superficial na formação da placa microbiana é bem estabelecida na literatura, havendo uma correlação positiva entre rugosidade e aderência de microrganismos (Hannig ${ }^{16}$ 1999, Morgan e Wilson ${ }^{24}$ 2001, Quirinem e Bollen ${ }^{35}$ 1995, Radford et al. ${ }^{38}$ 1998, Zissis et al..$^{59}$ 2000). Como as resinas acrílicas autopolimerizáveis e termopolimerizáveis apresentam rugosidade suficiente para promover a aderência microbiana, independentemente do acabamento utilizado, (Zissis et al..$^{59}$ 2000), cuidados na obtenção das próteses devem ser tomados. As superfícies não polidas devem ser obtidas a partir da prensagem sobre modelos vazados com gesso de alta qualidade, de forma que não sejam produzidas maiores porosidades e, consequentemente, maiores irregularidades nas superfícies da resina acrílica (Monsenego ${ }^{22}$ 2000) Além disso, o polimento das superfícies externas da prótese é considerado de extrema importância para o controle da placa microbiana, uma vez que as superfícies polidas facilitam a higienização da prótese e dificultam a aderência de microrganismos (Yamauchi et al. ${ }^{58}$ 1990). O polimento das próteses em laboratório propicia a menor rugosidade superficial que o polimento clínico obtido com kits de silicone (Braun et al. ${ }^{6} 2003$, Hannig ${ }^{16}$ 1999, O’Donnell et al. ${ }^{30}$ 2003, Quirinen et al. ${ }^{36}$ 1990). A energia livre de superfície também 
Neppelenbroek KH, Pinto ECT, Pavarina AC, Vergani CE, Jorge JH, Almilhatti HJ. Aderência de microorganismos em materiais para base de próteses Revista de Odontologia da Universidade Cidade de São Paulo 2009 mai-ago; 21(2): 126-36

pode influenciar a aderência microbiana, sendo esperada menor força de adesão dos microrganismos nas superfícies com baixa energia. Quirinen et al. ${ }^{36} 1990$, Schakenraad et al. ${ }^{41}$ 1986). Além disso, tem sido demonstrado que os microrganismos com baixa energia de superfície aderem preferencialmente às superfícies sólidas com baixa energia livre (Absolon et al. ${ }^{1}$ 1983), Assim, a adesão dos microrganismos segundo a energia de superfície é em parte influenciada pelo tipo de material utilizado para confecção das próteses (substrato) e em parte pela qualidade da microbiota bucal do indivíduo, sobretudo aquela relacionada à película original formada após o contato das próteses com a saliva do paciente (Quirinen et al. ${ }^{36} 1990$, Schakenraad et al. ${ }^{41}$ 1986). Entretanto, estudos têm sugerido que a rugosidade superficial é significativamente mais relevante para aderência microbiana que a energia livre de superfície (Hannig ${ }^{16}$ 1999, Quirinen et al. ${ }^{36}$ 1990). Outro fator que pode interferir na aderência de microrganismos é a umectabilidade do substrato (Monsénégo et al. ${ }^{23}$ 1989). É provável que nas superfícies com maior umectabilidade ocorra maior absorção das proteínas salivares, facilitando, dessa forma, a aderência dos microrganismos. Entretanto, tem sido observado que as resinas reembasadoras à base de silicone apresentam maior aderência que as resinas termopolimerizáveis. Assim, é possível que a umectabilidade do substrato influencie de forma menos significativa a aderência microbiana (Quirinem e Bollen ${ }^{35}$ 1995, Quirinen et al. ${ }^{36}$ 1990). No entanto, essa característica, bem como outras propriedades superficiais das resinas para base de prótese e sua relação com a aderência microbiana, ainda precisam ser avaliadas.

Tem sido também relatado que a qualidade das proteínas salivares formadoras do biofilme é mais importante na aderência microbiana que as características superficiais das resinas acrílicas (Nikawa et al. ${ }^{28}$ 1998). Portanto, além do tipo de material utilizado na confecção das próteses, é necessário considerar a qualidade da microbiota do paciente. Essa é influenciada por diversas condiçóes, como dieta, qualidade da saliva, condiçôes sistêmicas e uso de medicamentos. A dieta rica em carboidratos favorece a aderência de microrganismos, sobretudo os relacionados ao desenvolvimento de cáries dentais (Nikawa et al. ${ }^{29}$ 1999). Como esses fatores são de difícil controle, cabe ao profissional instruir adequadamente os métodos de limpeza e desinfecção das próteses para que haja um controle efetivo do biofilme presente nelas (Kulak et al. ${ }^{18}$ 1997, Neppelenbroek et al. ${ }^{26}$ 2008, Nepellenbroek et al. ${ }^{27}$ 2003, Silva et al. ${ }^{43}$ 2006, Webb et al..$^{54}$ 2005). Nesse contexto, ênfase deve ser dada à associação da escovação das próteses com dentifrício, seguida por desinfecção em microondas (Neppelenbroek et al. ${ }^{26}$ 2008, Nepellenbroek et al. ${ }^{27}$ 2003, Silva et al. ${ }^{43}$ 2006). Indivíduos que fazem uso de medicamentos que induzem xerostomia são mais propensos a apresentar placa microbiana nas próteses e infecçóes bucais, uma vez que a açãos imunológica, de limpeza e de proteção a traumas da saliva é reduzida (Massad e Cagna ${ }^{21}$ 2002, Torres et al. ${ }^{49}$ 2002). Pacientes que fazem uso de medicamentos imunossupressores e indutores de hiposalivação ou que apresentam doenças sistêmicas que comprometam o sistema imunológico são mais susceptíveis a apresentar microrganismos oportunistas na sua microbiota (Pfaller e Welzel ${ }^{31}$ 1992, Pittet el al. ${ }^{33} 1993$, Willis et al. ${ }^{56} 2000$, Wingard ${ }^{57}$ 1995). Esses pacientes são geralmente idosos e, na maioria, mulheres, que também sofrem alteraçôes hormonais após a menopausa (Pires et al. ${ }^{32}$ 2002, Ritchie et al. ${ }^{39}$ 1969). A utilização contínua das próteses, inclusive no período noturno, também favorece o acúmulo de placa, uma vez que as próteses ficam em contato com os tecidos bucais quando há um menor nível de salivação (Massad e Cagna $^{21}$ 2002, Nikawa et al. ${ }^{28}$ 1998). Além disso, as próteses, especialmente as totais superiores, podem traumatizar os tecidos de suporte pela pressão desenvolvida com a retenção e, ainda, reduzir ainda mais a salivação pela oclusão dos ductos das glândulas salivares secundárias (Lombardi e Budtz-Jorgesen ${ }^{19}$ 1993). A perda da adaptação das próteses aos tecidos de suporte ao longo dos anos de uso também gera neles trauma contínuo, o que os torna mais propensos a infecções (Lombardi e Budtz-Jorgesen ${ }^{19}$ 1993, Ship et al. ${ }^{42}$ 2002). A necessidade de reembasamento ou substituição das próteses deve ser avaliada sempre pelo profissional na fase de controle posterior (Lombardi e Budtz-Jorgesen ${ }^{19}$ 1993, Ritchie et al. ${ }^{39}$ 1969).

O profissional deve reconhecer a importância dos fatores envolvidos na aderência microbiana e, na medida do possível, controlá-los quer seja pela escolha de materiais apropriados para confecção e reembasamento das próteses e de cuidados durante sua obtenção quer seja pela adequada motivaçáo do paciente para uso e higienização adequada de suas próteses removíveis, totais ou parciais. 


\section{CONCLUSÕES}

A aderência dos microrganismos aos materiais para base de prótese é complexa, sendo influenciada por diversos fatores:

- Técnica de polimerização e composição das resinas acrílicas;

- Características superficiais dos materiais para base de prótese;

- Tipo e forma dos microrganismos;

- Técnicas de higienização e período de utilização das próteses ;

- Vida útil e condiçóes de adaptação das próteses;

- Condiçóes da saúde bucal e geral do paciente.

\section{REFERÊNCIAS}

1. Absolom DR, Lamberti FV, Policova Z, Zingg W, van Oss CJ, Neumann AW. Surface thermodynamics of bacterial adhesion. ApplEnviron Microbiol 1983 Jul;46(1):90-7.

2. Arima T, Murata H, Hamada T. Properties of highly cross-linked autopolymerizing reline acrylic resins. J Prosthet Dent 1995 Jan; 73(1):55-9.

3. Baena-Monroy T, Moreno-Maldonado V, FrancoMartínez F, Aldaipe-Barrios B, Quindós G, SanchesVargas LO. Candida albicans, Staphylococcus aureus and Streptococcus mutans colonization in patients wearing dental prosthesis. Med Oral Patol Oral Cir Bucal 2005 Apr 1; 10 Suppl 1:E27-39.

4. Barnabé W, de Mendonça Neto T, Pimenta FC, Pegoraro LF, Scolaro JM. Efficacy of sodium hypochlorite and coconut soap used as disinfecting agents in reduction of denture stomatitis, Streptococcus mutans and Candida albicans. J Oral Rehabil 2004 May; 31(5):453-9.

5. Baysan A, Whiley R, Wright PS. Use of microwave energy to disinfect a long-term soft lining material contaminated with Candida albicans or Staphylococcus aureus. J Prosthet Dent 1998 Apr; 79(4):454-8.

6. Braun KO, Mello JA, Rached RN, Del Bel Cury AA. Surface texture and some properties of acrylic resins submitted to chemical polishing. J Oral Rehabil 2003 Jan; 30(1):91-8.

7. Budtz-Jörgensen E. Clinical aspects of Candida infection in denture wearers. J Am Dent Assoc 1978 Mar; 96(3):474-9.

8. Budtz-Jørgensen E. Materials and methods for cleaning dentures. J Prosthet Dent 1979 Dec; 42(6):619-23.
9. Bunch J, Johnson GH, Brudvik JS. Evaluation of hard direct reline resins. J Prosthet Dent 1987 Apr; 57(4):512-9.

10. Busscher HJ, Weerkamp AH, van der Mei HC, van Pelt AW, de Jong HP, Arends J. Measurement of the surface free energy of bacterial cell surfaces and its relevance for adhesion. Appl Environ Microbiol 1984 Nov; 48(5):980-3.

11. Chau VB, Saunders TR, Pimsler M, Elfring DR. Indepth disinfection of acrylic resins. J Prosthet Dent 1995 Sep; 74(3):309-13.

12. Compagnoni MA, Barbosa DB, Souza RF, Pero AC. The effect of polymerization cycles on porosity of microwave-processed denture base resin. J Prosthet Dent 2004 Mar; 91(3):281-5.

13. Cross LJ, Williams DW, Sweeney CP, Jackson MS, Lewis MA, Bagg J. Evaluation of the recurrence of denture stomatitis and Candida colonization in a small group of patients who received itraconazole. Oral Surg Oral Med Oral Pathol Oral Radiol Endod 2004 Mar; 97(3):351-8.

14. Davenport JC. The oral distribution of candida in denture stomatitis. Br Dent J 1970 Aug 18; 129(4):151-6.

15. Gristina AG, Hobgood CD, Webb LX, Myrvik QN. Adhesive colonization of biomaterials and antibiotic resistance. Biomaterials. 1987 Nov; 8(6):423-6.

16. Hannig M. Transmission electron microscopy of early plaque formation on dental materials in vivo. Eur J Oral Sci. 1999 Feb; 107(1):55-64.

17. Kuhar M, Funduk N. Effects of polishing techniques on the surface roughness of acrylic denture base resins. J Prosthet Dent 2005 Jan; 93(1):76-85. 
Neppelenbroek KH, Pinto ECT, Pavarina AC, Vergani CE, Jorge JH, Almilhatti HJ. Aderência de microorganismos em materiais para base de próteses Revista de Odontologia da Universidade Cidade de São Paulo 2009 mai-ago; 21(2): 126-36

18. Kulak Y, Arikan A, Kazazoglu E. Existence of Candida albicans and microorganisms in denture stomatitis patients. J Oral Rehabil 1997 Oct; 24(10):788-90.

19. Lombardi T, Budtz-Jörgensen E. Treatment of denture-induced stomatitis: a review. Eur J Prosthodont Restor Dent 1993 Sep; 2(1):17-22.

20. Loney RW, Moulding MB, Hacker CH, Ritsco RG. Finishing and polishing of a poly (fluoroalkoxyphosphazene) resilient denture liner Int J Prosthodont 1994 Jul-Aug; 7(4):362-7.

21. Massad JJ, Cagna DR. Removable prosthodontic therapy and xerostomia. Treatment considerations. Dent Today 2002 Jun; 21(6):80-2, 84, 86-7.

22. Monsenego P. Presence of microorganisms on the fitting denture complete surface: study 'in vivo'. $J$ Oral Rehabil 2000 Aug; 27(8):708-13.

23. Monsénégo P, Baszkin A, Costa ML, Lejoyeux J. Complete denture retention. Part II: Wettability studies on various acrylic resin denture base materials. J Prosthet Dent 1989 Sep; 62(3):308-12.

24. Morgan TD, Wilson M. The effects of surface roughness and type of denture acrylic on biofilm formation by Streptococcus oralis in a constant depth film fermentor. J Appl Microbiol 2001 Jul; 91(1):4753.

25. Neppelenbroek KH, Mima EGO, Spolidorio DMP, Giampaolo ET, Vergani CE, Pavarina AC. Efetividade da irradiação por microondas na desinfecção de resinas reembasadoras rígidas e resina acrílica para base de prótese. Rev Odontol UNESP 2006 outdez 35(4): 303-11.

26. Neppelenbroek KH, Pavarina AC, Palomari Spolidorio DM, Sgavioli Massucato EM, Spolidorio LC, Vergani CE. Effectiveness of microwave disinfection of complete dentures on the treatment of Candida-related denture stomatitis. J Oral Rehabil 2008 Nov; 35(11): 836-46.

27. Neppelenbroek KH, Pavarina AC, Spolidorio DM, Vergani CE, Mima EG, Machado AL. Effectiveness of microwave sterilization on three hard chairside reline resins. Int J Prosthodont 2003 Nov-Dec;16(6):61620.

28. Nikawa H, Hamada T, Yamamoto T. Denture plaque--past and recent concerns. J Dent 1998 May; 26(4):299-304.
29. Nikawa H, Hamada T, Yamashiro H, Kumagai $H$. A review of in vitro and in vivo methods to evaluate the efficacy of denture cleansers. Int J Prosthodont 1999 Mar-Apr; 12(2):153-9.

30. O’Donnell EF, Radford DR, Sinclair GF, Clark RK. Chairside polishing of heat-cured acrylic resin: an SEM and EDA study. Int J Prosthodont 2003 May-Jun; 16(3):233-8.

31. Pfaller M, Wenzel R. Impact of the changing epidemiology of fungal infections in the 1990s. Eur J Clin Microbiol Infect Dis 1992 Apr; 11(4):287-91.

32. Pires FR, Santos EB, Bonan PR, De Almeida OP, Lopes MA. Denture stomatitis and salivary Candida in Brazilian edentulous patients. J Oral Rebabil 2002 Nov; 29(11):1115-9.

33. Pittet D, Li N, Wenzel RP. Association of secondary and polymicrobial nosocomial bloodstream infections with higher mortality. Eur J Clin Microbiol Infect Dis 1993 Nov;12(11):813-9.

34. Quirynen M, Marechal M, Busscher HJ, Weerkamp $\mathrm{AH}$, Arends J, Darius PL, van Steenberghe D. The influence of surface free-energy on planimetric plaque growth in man. J Dent Res 1989 May; 68(5):796-9.

35. Quirynen M, Bollen CM. The influence of surface roughness and surface-free energy on supra- and subgingival plaque formation in man. A review of the literature. J Clin Periodontol 1995 Jan; 22(1):1-14.

36. Quirynen M, Marechal M, Busscher HJ, Weerkamp AH, Darius PL, van Steenberghe D. The influence of surface free energy and surface roughness on early plaque formation. An in vivo study in man. $J$ Clin Periodontol 1990 Mar; 17(3):138-44.

37. Radford DR, Challacombe SJ, Walter JD. Denture plaque and adherence of Candida albicans to denturebase materials in vivo and in vitro. Crit Rev Oral Biol Med 1999; 10(1):99-116.

38. Radford DR, Sweet SP, Challacombe SJ, Walter JD. Adherence of Candida albicans to denture-base materials with different surface finishes. J Dent 1998 Sep; 26(7):577-83.

39. Ritchie GM, Fletcher AM, Main DM, Prophet AS. The etiology, exfoliative cytology, and treatment of denture stomatitis. J Prosthet Dent 1969 Aug; 22(2):185-200. 
40. Sato $M$, Tsuchiya $H$, Akagiri $M$, Takagi $N$, Iinuma M. Growth inhibition of oral bacteria related to denture stomatitis by anti-candidal chalcones. Aust Dent J 1997 Oct; 42(5):343-6.

41. Schakenraad JM, Busscher HJ, Wildevuur CR, Arends J. The influence of substratum surface free energy on growth and spreading of human fibroblasts in the presence and absence of serum proteins. $J$ Biomed Mater Res 1986 Jul-Aug; 20(6):773-84.

42. Ship JA, Pillemer SR, Baum BJ. Xerostomia and the geriatric patient. J Am Geriatr Soc 2002 Mar; 50(3):535-43.

43. Silva MM, Vergani CE, Giampaolo ET, Neppelenbroek KH, Spolidorio DM, Machado AL. Effectiveness of microwave irradiation on the disinfection of complete dentures. Int J Prosthodont 2006 May-Jun; 19(3):288-93.

44. Sipahi C, Anil N, Bayramli E. The effect of acquired salivary pellicle on the surface free energy and wettability of different denture base materials. J Dent 2001 Mar; 29(3):197-204.

45. Smith LT, Powers JM, Ladd D. Mechanical properties of new denture resins polymerized by visible light, heat, and microwave energy. Int J Prosthodont 1992 Jul-Aug; 5(4):315-20.

46. Sofou A, Emmanouil J, Peutzfeldt A, Owall B. The effect of different polishing techniques on the surface roughness of acrylic resin materials. Eur J Prosthodont Restor Dent 2001 Sep-Dec;9(3-4):117-22.

47. Tari BF, Nalbant D, Dogruman Al F, Kustimur S. Surface roughness and adherence of Candida albicans on soft lining materials as influenced by accelerated aging. J Contemp Dent Pract 2007 Jul 1; 8(5):18-25.

48. Theilade E, Budtz-Jørgensen E. Predominant cultivable microflora of plaque on removable dentures in patients with denture-induced stomatitis. Oral Microbiol Immunol 1988 Mar; 3(1):8-13.

49. Torres SR, Peixoto CB, Caldas DM, Silva EB, Akiti T, Nucci $\mathrm{M}$ et al. Relationship between salivary flow rates and Candida counts in subjects with xerostomia. Oral Surg Oral Med Oral Pathol Oral Radiol Endod 2002 Feb; 93(2):149-54.
50. Umemoto K, Kurata S. Basic study of a new denture base resin applying hydrophobic methacrylate monomer. Dent Mater J 1997 Jun;16(1): 21-30.

51. Van Pelt AW, Weerkamp AH, Uyen MH, Busscher HJ, de Jong HP, Arends J. Adhesion of Streptococcus sanguis $\mathrm{CH} 3$ to polymers with different surface free energies. Appl Environ Microbiol 1985 May; 49(5):1270-5.

52. Verran J, Maryan CJ. Retention of Candida albicans on acrylic resin and silicone of different surface topography. J Prosthet Dent 1997 May; 77(5):535-9.

53. Waters MG, Williams DW, Jagger RG, Lewis MA. Adherence of Candida albicans to experimental denture soft lining materials. J Prosthet Dent 1997 Mar; 77(3):306-12.

54. Webb BC, Thomas CJ, Whittle T. A 2-year study of Candida-associated denture stomatitis treatment in aged care subjects. Gerodontology 2005 Sep; 22(3):16876.

55. Weerkamp AH, van der Mei HC, Busscher HJ. The surface free energy of oral streptococci after being coated with saliva and its relation to adhesion in the mouth. J Dent Res 1985 Oct; 64(10):1204-10.

56. Willis AM, Coulter WA, Sullivan DJ, Coleman DC, Hayes JR, Bell PM et al. Isolation of C. dubliniensis from insulin-using diabetes mellitus patients. J Oral Pathol Med 2000 Feb; 29(2):86-90.

57. Wingard JR. Importance of Candida species other than $C$. albicans as pathogens in oncology patients. Clin Infect Dis 1995 Jan; 20(1):115-25.

58. Yamauchi M, Yamamoto K, Wakabayashi M, Kawano J. In vitro adherence of microorganisms to denture base resin with different surface texture. Dent Mater J 1990 Jun;9(1):19-24.

59. Zissis AJ, Polyzois GL, Yannikakis SA, Harrison A. Roughness of denture materials: a comparative study. Int J Prosthodont 2000 Mar-Apr; 13(2):136-40.

Recebido em: 16/09/2008

Aceito em: 1/06/2009 